\title{
THE DWARFS BEYOND: THE STELLAR-TO-HALO MASS RELATION FOR A NEW SAMPLE OF INTERMEDIATE REDSHIFT LOW-MASS GALAXIES
}

\author{
Sarah H. Miller ${ }^{1,2,3}$, Richard S. Ellis ${ }^{1}$, Andrew B. Newman ${ }^{1,4}$, And Andrew Benson ${ }^{4}$ \\ ${ }^{1}$ California Institute of Technology, 1200 E. California Blvd, Pasadena, CA 91125, USA; smiller@ astro.caltech.edu \\ ${ }^{2}$ University of California - Riverside, 900 University Ave, Riverside, CA 92521, USA \\ ${ }^{3}$ University of California - Irvine, Irvine, CA 92697, USA \\ ${ }^{4}$ Carnegie Observatories, 813 Santa Barbara St, Pasadena, CA 91101, USA \\ Received 2013 October 2; accepted 2014 January 7; published 2014 February 4
}

\begin{abstract}
A number of recent challenges to the standard $\Lambda \mathrm{CDM}$ paradigm relate to discrepancies that arise in comparing the abundance and kinematics of local dwarf galaxies with the predictions of numerical simulations. Such arguments rely heavily on the assumption that the Local Volume's dwarf and satellite galaxies form a representative distribution in terms of their stellar-to-halo mass ratios. To address this question, we present new, deep spectroscopy using DEIMOS on Keck for 82 low-mass $\left(10^{7}-10^{9} M_{\odot}\right)$, star-forming galaxies at intermediate redshift $(0.2<z<1)$. For $50 \%$ of these we are able to determine resolved rotation curves using nebular emission lines and thereby construct the stellar mass Tully-Fisher relation to masses as low as $10^{7} M_{\odot}$. Using scaling relations determined from weak lensing data, we convert this to a stellar-to-halo mass relation for comparison with abundance matching predictions. We find a discrepancy between our observations and the predictions from abundance matching in the sense that we observe 3-12 times more stellar mass at a given halo mass. We suggest possible reasons for this discrepancy, as well as improved tests for the future.
\end{abstract}

Key words: galaxies: dwarf - galaxies: evolution - galaxies: fundamental parameters - galaxies: kinematics and dynamics

Online-only material: color figures

\section{INTRODUCTION}

The $\Lambda$-dominated cold dark matter model $(\Lambda \mathrm{CDM})$ has been remarkably successful in the interpretation of the largescale structure of the universe and its evolution as probed by observations of the cosmic microwave background, the local galaxy distribution, and independent probes of the dark matter power spectrum such as the Ly $\alpha$ forest and weak gravitational lensing. However, significant challenges remain when the theory is confronted with observations on galaxy scales (see the review by Weinberg et al. 2013).

A long-standing question is the apparent mismatch of the abundance of visible satellites in the Milky Way halo compared to that predicted from the steep dark matter power spectrum (Klypin et al. 1999; Moore et al. 1999). Possible explanations include the likelihood that halos are dark due to the early photoionizing background (Bullock et al. 2000), energetic feedback which suppressed growth (Font et al. 2011), or an observational bias whereby many satellites have yet to be discovered, either because they are hidden by the Galactic plane or are too faint for existing surveys (Tollerud et al. 2008). Recently, a more fundamental challenge relates to a surprising discrepancy between the observed and expected maximum circular velocities ( $\left.V_{\max }\right)$ for the most massive Local Group satellites. The best-studied dwarf spheroidals near the Milky Way have $12<V_{\max }<25 \mathrm{~km} \mathrm{~s}^{-1}$, whereas the Aquarius (Springel et al. 2008) and the Via Lactea II simulations (Diemand et al. 2007, 2008) predict at least 10 subhalos should be visible with $V_{\max }>25 \mathrm{~km} \mathrm{~s}^{-1}$ (Boylan-Kolchin et al. 2011). These simulations predict DM halos five times more massive than what could be inferred given the observed densities of the satellite dwarf spheroidals. No incontrovertible explanation currently satisfies the "Too Big to Fail" (Boylan-Kolchin et al.
2011) problem; some consider it a result of poorly understood baryonic feedback effects (e.g., Pontzen \& Governato 2012), whereas others postulate a fundamental departure from the current dark matter model (e.g., Rocha et al. 2013).

Both of the interrelated problems above rely inescapably on the question of the whether the Milky Way and its satellites are representative of those typical of the larger cosmic volumes. Furthermore, predicting satellite properties of the Milky Way is complicated by the difficulty in inferring its halo mass. For dwarf galaxies and satellites, the halo mass range $10^{7} M_{\odot}<$ $M<10^{9} M_{\odot}$, where resolved kinematic data are sparse, is particularly interesting. A current inventory of directly measured rotation curves consists of the Magellanic Clouds and nine of the brightest satellites of the Milky Way. The HI Nearby Galaxy Survey contributes a further four galaxies (Oh et al. 2011) to the eight gas-rich systems from Stark et al. (2009). Many more early-type dwarf galaxies can be observed in the dense regions of the local universe which were probably once small disk galaxies before being transformed to their present state via environmental processes (Kormendy \& Bender 2012). The consensus in the literature is that $\mathrm{dE} / \mathrm{dS} 0$ galaxies in the stellar mass range of $10^{7}$ and $10^{9} M_{\odot}$ do not possess very massive dark halos, which does not agree with predictions by cosmological simulations (De Rijcke et al. 2006; Chilingarian et al. 2008).

A more general way to view this problem is the relationship between the stellar mass and halo mass (the so-called SHM relation). This relation is well-constrained down to stellar masses of $10^{9} M_{\odot}$ by various methods, including weak lensing (e.g., Mandelbaum et al. 2006; Leauthaud et al. 2012), abundance matching (e.g., Moster et al. 2010; Behroozi et al. 2010), and stellar kinematics (e.g., Conroy et al. 2007; More et al. 2011). The most effective probe for lower-mass systems is resolved dynamics for rotationally supported systems, i.e., the stellar mass 
Tully-Fisher (TF) relation (e.g., Geha et al. 2006; Pizagno et al. 2007). Various methods can be used to relate observed velocities to the halo virial circular velocity, which we discuss further as motivation for this work in the following section.

This paper presents results from a new observational program whose goal is to extend the stellar mass TF relation into the relevant mass range $10^{7} M_{\odot}<M_{*}<10^{9} M_{\odot}$ at intermediate redshifts, and constrain the stellar-to-halo mass (SHM) relation well beyond the confines of the Local Group. As we demonstrate here, such an observational program to investigate the dwarf density discrepancy described here is now feasible.

An organization of the paper is as follows. In Section 2 we describe our motivation in evaluating the SHM relation in the dwarf regime. In Section 3, we describe the selection criteria for our sample, the DEIMOS spectroscopic data, and the resolved photometry from the Hubble Space Telescope (HST) from which our stellar mass estimates are derived. Section 3.4 specifically discusses the techniques we use to fit kinematic models to our low-mass galaxies. In Section 4, we present our stellar mass TF relation and introduce our method for converting this relation to the required SHM relation. In Section 5, we discuss our SHM results in the context of predictions from $\Lambda \mathrm{CDM}$ cosmological simulations and abundance matching methods. Throughout the paper, we adopt a $\Omega_{\Lambda}=0.7, \Omega_{m}=0.3, H_{0}=70 \mathrm{~km} \mathrm{~s}^{-1} \mathrm{Mpc}^{-1}$ cosmology. All magnitudes refer to the AB system.

\section{MOTIVATION}

The methods used for relating velocities measured across the optical extent of galaxies to the halo virial circular velocity, or $V_{200}$ (e.g., Navarro et al. 1997; Bullock et al. 2001; Seljak 2002; Eke et al. 2006; Dutton et al. 2010; Reyes et al. 2012), have not been calibrated directly in the dwarf regime. Yet if the relation of Reyes et al. (2012) $)^{5}$ to convert optical velocities to halo velocities is extrapolated to low mass, there is a serious discrepancy between the resulting halo circular velocities and those calculated based on abundance matching techniques for dwarf galaxies $<10^{9} M_{\odot}$ (Figure 1$)$.

A key question is whether this offset is a generic result for low-mass dwarf galaxies beyond the Local Group. Furthermore, how do the Local Group galaxies relate to the full scatter in the SHM relation of a larger, more representative sample? Although the conversion from dynamical mass to halo mass will require careful evaluation, given such scaling relations are currently only determined for higher mass systems than the focus of this work, data can hopefully provide the basis for future comparisons with numerical simulations. Nevertheless, while extrapolation of Reyes et al. (2012) is uncertain, recent simulation work supporting the Next Generation Virgo Cluster Survey suggests this extrapolation is reasonably accurate (J. Grossauer et al., in preparation).

\section{DATA}

We present a new spectroscopic data set which exploits the significant multiplex gain of DEIMOS to address the kinematic properties of low-mass, star-forming dwarf galaxies

\footnotetext{
5 In Reyes et al. (2012), the optical-to-virial velocity ratios are derived from both directly measured velocities from rotation curves along with $V_{200}$ values from halo masses derived with galaxy-galaxy weak lensing. A consistent and calibrated selection was used between samples rotation curve and weak lensing samples, and the constructed TF relations are consistent between their work at $z \sim 0$ and our previous work (Miller et al. 2011) with the same mass range at higher redshift.
}

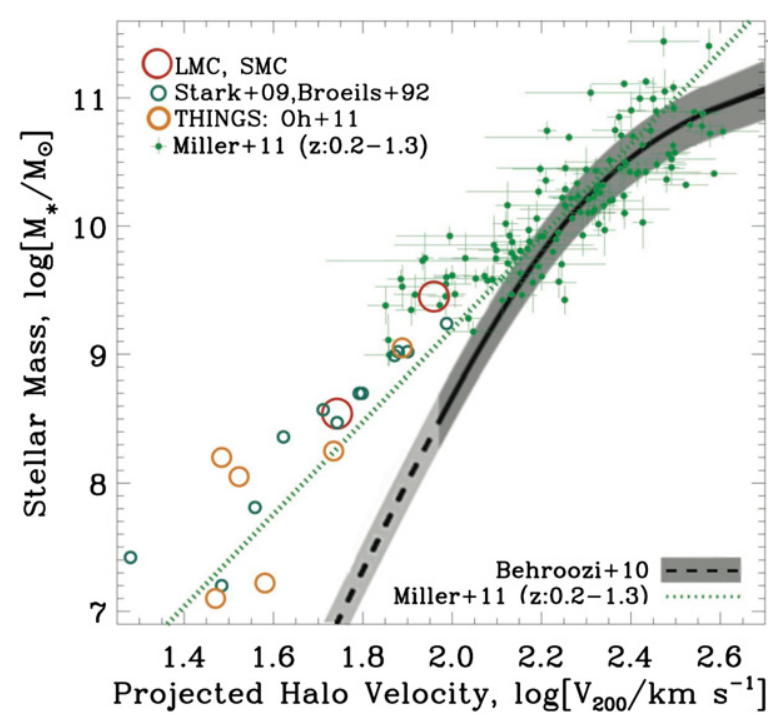

Figure 1. Motivating this work, there appears to be a divergence between the abundance matching result and the stellar mass Tully-Fisher relation, which has been converted to halo velocity $V_{200}$ using the extrapolated Reyes et al. (2012) relation between observed velocities as a function of stellar mass and $V_{200}$. Also plotted are the converted velocities of local volume galaxies, which further suggest a discrepancy between observed velocities and abundance matching, however, the scatter in the stellar-to-halo mass relation is unclear with so few data points.

(A color version of this figure is available in the online journal.)

at intermediate redshift $(0.2<z<1.0)$. Two advances make it practical to target dwarf galaxies at intermediate redshifts. The first is our demonstrated ability to recover rotational velocities on small angular scales from our earlier work on high redshift disk galaxies (Miller et al. 2011, 2012), the relevant techniques of which we describe further in Section 3.4. The second is the availability of remarkably deep HST Wide-Field Camera 3 (WFC3) infra-red imaging from the Cosmic Assembly NearIR Deep Extragalactic Legacy Survey (CANDELS; Grogin et al. 2011; Koekemoer et al. 2011), enabling us to conduct resolved photometric models of dwarf targets and construct catalogs (i.e., Newman et al. 2013) of photometric redshifts and stellar mass estimates along with deep optical Subaru/ XMM-Newton Deep Survey data for much lower mass systems at intermediate redshifts than previously possible. Although a $10 \mathrm{~m}$ class telescope and long exposures are necessary to study these low-mass sources efficiently, as the target density within a DEIMOS field is substantial, the multiplex gain offers a huge advantage over modest samples of dwarf galaxies studied individually. Targets of similar mass available from the Sloan Digital Sky Survey have a much lower surface density and cannot be efficiently surveyed with the current multiplex gains on 4 or $10 \mathrm{~m}$ aperture telescopes.

\subsection{Sample Selection and DEIMOS Observations}

In this work, we examine a large number of low-mass galaxies at intermediate redshift drawn from the UKIRT Ultradeep Survey field (UDS) using the photometric catalog derived by Newman et al. (2013). We adopted a magnitude limit of $i_{A B}=25$ which corresponds approximately to a median stellar mass limit of $10^{7.2}, 10^{8.0}$, and $10^{8.5} M_{\odot}$ for $z \simeq 0.2,0.5$, and 0.8 , respectively (Figure 2). Two further criteria were used to select targets, a photometric redshift range $0.2<z<0.8$ and a stellar mass range $\log \left(M_{*} / M_{\odot}\right)<9.3$. Photometric redshifts were determined using the EAzY code (Brammer et al. 2008) and 


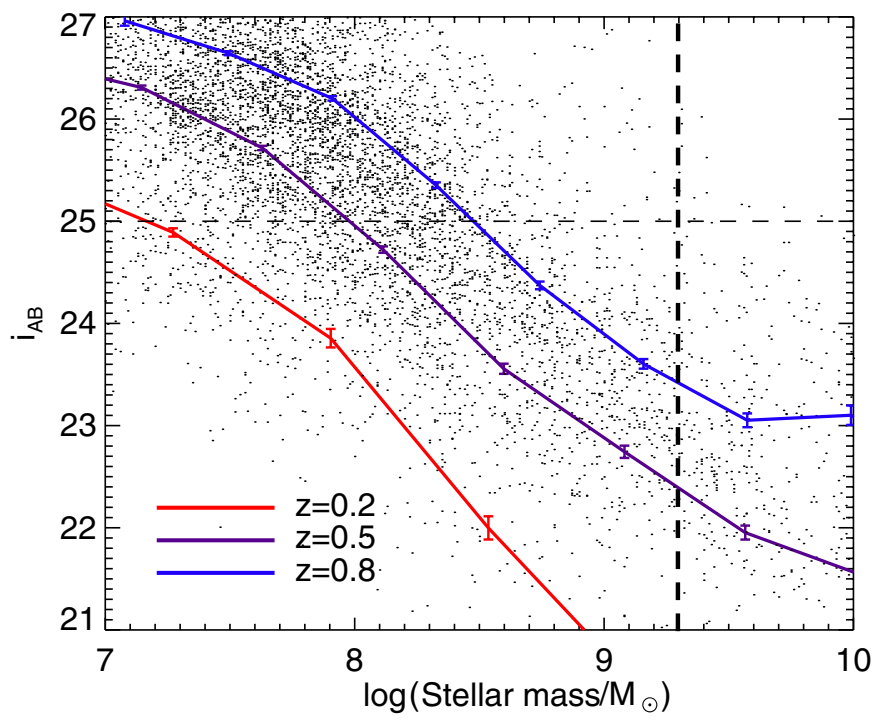

Figure 2. Median magnitude curves within the Newman et al. (2013) catalog as a function of apparent $i_{A B}$ magnitude for various redshifts of interest. For the redshift range $0.2<z<0.8$, galaxies with stellar masses as low as $10^{7.2}$ to $10^{8.5} M_{\odot}$, respectively, can be identified, thereby sampling the important range of the stellar-to-halo mass relation. Dashed lines denote the magnitude and stellar mass limits used in preparing the DEIMOS target list.

(A color version of this figure is available in the online journal.)

stellar masses were estimated using the FAST code (Kriek et al. 2009)_for more details, see Section 3.2. In constructing the multislit DEIMOS mask, slitlets were aligned to the major axes for significantly inclined targets (based on photometry using SExtractor; Bertin \& Arnouts 1996). The mask position angle was selected so as to optimally fill that portion of the UDS field with available WFC3 coverage.

We used the $12001 \mathrm{~mm}^{-1}$ grating in DEIMOS centered at $7500 \AA$ so that $[\mathrm{O}$ III] and $\mathrm{H} \beta$ line emission would be sampled for the full targeted redshift range, $\mathrm{H} \alpha$ emission for $z \simeq 0.2-0.4$ and [O II] for $z<0.6$. With $\sim 30 \mathrm{~km} \mathrm{~s}^{-1}$ kinematic resolution, our earlier work demonstrated the ability to recover rotation curves with characteristic velocities of $50 \mathrm{~km} \mathrm{~s}^{-1}$ (Miller et al. 2011, 2012). Our modeling code was well-tested using simulated data to rotational velocities as low as $30 \mathrm{~km} \mathrm{~s}^{-1}$. This corresponds to the characteristic maximal velocity for dwarf galaxies with stellar masses of $<10^{6} M_{\odot}$ in the abundance matching method, but to a stellar mass of $10^{7.3} M_{\odot}$ in the extrapolated stellar mass TF relation of Miller et al. (2011). Although our typical targets are only $1.5-2^{\prime \prime}$ across, this corresponds to the extent of more massive systems successfully targeted at $z>1$ using our code (Miller et al. 2012, 2013).

We successfully secured $10 \mathrm{hr}$ of science-quality exposure time with the DEIMOS instrument on a single mask in the UDS field on 2012 December 11 and 12. A total of 82 dwarfs were targeted, but not all objects observed show ordered rotation or line emission. However, we do not seek to construct a complete sample via this approach; of course, such completeness is not even possible in local data. In summary, we find that $28 \%$ $(N=23)$ of the targeted sample reveal no significant line emission. These sources occupy the lower surface brightness portion of the photometric distribution suggesting their line emission may simply be too faint to be detectable. We find $22 \%$ $(N=18)$ have unresolved line emission precluding any attempt to construct rotation curves. The remaining $50 \%(N=41)$ have resolved emission from which we can attempt to derive rotation curves (see Figure 3). This mix of target properties is actually

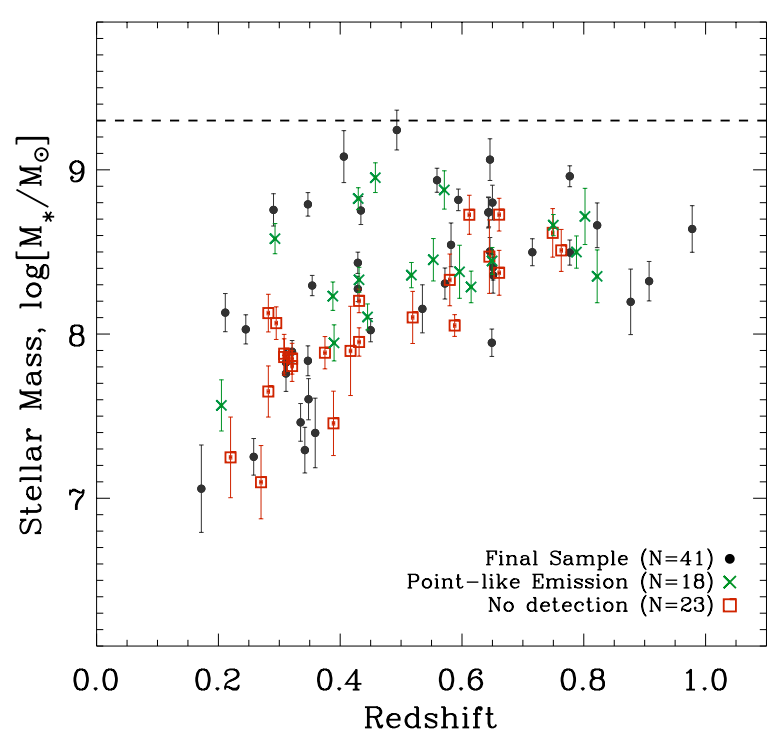

Figure 3. Stellar mass and redshift distribution for the 82 dwarf galaxies studied spectroscopically. Twenty-three galaxies which revealed no significant line emission are plotted as red boxes (in the colored version) at their photometric redshifts. Eighteen galaxies which revealed unresolved line emission are denoted with green crosses (in the colored version) at their spectroscopic redshift. The other 41 galaxies show resolved line emission are denoted with black circular points. The dashed line marks the stellar mass limit for the overall sample.

(A color version of this figure is available in the online journal.)

similar to that recovered in our previous higher mass samples at intermediate redshifts.

\subsection{Stellar Mass Estimates}

Stellar mass estimates were derived using the FAST (Fitting and Assessment of Synthetic Templates) code (Kriek et al. 2009) which fits stellar population synthesis templates (Bruzual \& Charlot 2003) to broadband photometry adopting a Chabrier (2003) initial mass function (IMF). For our photometric database (Newman et al. 2013), we use BVRiz from deep Subaru/XMM-Newton Deep Survey (Furusawa et al. 2008), with mosaics prepared by Cirasuolo et al. (2010); $J$ (F125W) and $H(\mathrm{~F} 160 \mathrm{~W})$ from CANDELS; and K photometry by the UKIDSS UDS Data Release 6 (DR6). We chose not to include photometry available at longer wavelengths due to blending confusion given the faintness of the sources targeted. We impose a floor of $\log (\tau / \mathrm{yr})=8.5$ for the timescale of the exponentially declining star formation histories given this appears appropriate for star-forming galaxies (e.g., Wuyts et al. 2011). Sub-solar metallicities of $Z=0.008,0.004$ as well as solar at 0.02 are permitted, consistent with the latest work on the metallicity of dwarf galaxies in the Local Group. FAST uses the Calzetti et al. (2000) reddening law. In summary the stellar population grid limits are: $\log (\tau / \mathrm{yr})=8.5-10.0 ; \log ($ age $/ \mathrm{yr})=7.0-10.1$; $A_{V}=0.0-3.0 ;$ metallicity $=0.004-0.02$.

We computed best-fitting model parameters with errors derived from Monte Carlo perturbations of the photometry, as well as the marginalized mean value and standard deviation of the parameter over the likelihood. We adopt the latter as they are more robust, however, there are marginal differences for this particular data set. We estimate the contribution of emission lines to the broad-band data to have an insignificant effect on the resulting stellar mass estimates, much smaller than the uncertainties.

It should be noted that the original set of stellar mass estimates used in the selection of the DEIMOS mask targets 
adopted a solar metallicity only, a Salpeter IMF, and the stellar population models of Coelho et al. (2007) which include more contribution from TP-AGB stars. Each of these choices is suited more to a higher mass sample than the dwarfs of this work. To compare to the final adopted estimates of this work, these alternate assumptions provided a systematic offset of +0.14 dex. For seven galaxies at stellar masses $\log \left(M_{*} / M_{\odot}\right)<7.7$ the results are particularly sensitive to these stellar population grid differences, but all estimates in the original set still remain below $\log \left(M_{*} / M_{\odot}\right)<8.7$.

Our stellar mass estimates adopt the same methods used in the state-of-the-art abundance matching methods for a consistent comparison to this work. Recent work has suggested that lowmass galaxy stellar mass estimates are likely underestimated when using exponential star-formation histories (A. Dominguez et al., in preparation). Burstier or more complex star formation histories may ultimately be found to be more realistic than the exponential histories adopted here, however, if we implemented such histories in this paper then we would lose the ability to compare directly with abundance matching methods.

\subsection{Image Measurements}

Examining the HST images of our sample, although most are small in angular extent (mean half-light radius, $\left\langle r_{1 / 2}\right\rangle \sim$ $1.2 \mathrm{kpc}$ ), the majority are regular in form with modest central concentrations. A high fraction have recognizable disks but few have prominent bulges.

We measure the radii and inclination of the dwarf galaxies in our sample assuming each galaxy is represented by a circular exponential disk. Scale radii and major-to-minor axis ratios are measured using GALFIT (Peng 2010) with initial estimate distributions based on SExtractor (Bertin \& Arnouts 1996) in the same Monte Carlo method as described in previous work (Miller et al. 2011, 2012, 2013). We adopted this formalism for consistency with work at higher masses, and particularly to extract velocities at a consistent location, namely at 2.2 times the scale radius, $r_{2.2}$ (Miller et al. 2011). The fiducial velocity measured at $r_{2.2}$ is denoted by $V_{2.2}$ and represents the peak rotational amplitude in a pure exponential disk. Although all of the dwarf galaxies of our sample are not necessarily best represented by a pure exponential, $V_{2.2}$ is generally well-suited in sampling the flattened portion of the rotation curve in galaxies (see Courteau 1997; Miller et al. 2011 for further discussion on this topic). For instance, allowing a free Sersic index fit in our sample produces a significantly more inconsistent set of effective radii by which to extract fiducial velocities for scaling relations.

While exponential disks are the simplest and most appropriate model for the morphologies of the majority of our dwarf sample, we also extract the velocity at a radius of $1 \mathrm{kpc}$ (see Table 1). These values are provided so that data samples analyzed with other techniques, namely predictions from numerical simulations, can more easily be compared to our values as exponential disk scale lengths via mock photometry measurements are not typically easily acquired.

\subsection{Dynamical Modeling}

The rotation curves for our sample were analyzed with the code developed in the previous studies of Miller et al. (2011, 2012, 2013). We adopt the arctangent functional form, and extract the velocity at 2.2 times the scale radius, $V_{2.2}$, correcting for the effect of inclination. This code is described and has been tested extensively for the effect of changes in scale/resolution and artificial redshifting in our earlier papers (Miller et al. 2011, 2012). It adopts an asymmetric Gaussian for the emission line profile of each spatial bin, and accounts for both spatially dependent velocity dispersion and surfacebrightness profiles of the traced emission line in the spectrum. Including these features, the code also accounts for the effects of blurring by atmospheric seeing in the spatial direction, and blurring by instrumental dispersion in the spectral direction. These are crucial ingredients for recovering the intrinsic velocity profile of galaxies, particularly those with small angular extents. Examples of our two-dimensional emission line spectra and model fits are given in Figure 4.

Table 1 lists all of the galaxies in our sample, including each of the derived physical properties, where possible, which have been described in Section 3.

\section{RESULTS}

To evaluate the SHM relation of our dwarf galaxy sample, we first consider the stellar mass TF relation (Figure 5), which represents the primary observational result of this paper, alongside earlier work on higher masses at intermediate redshift (Miller et al. 2011). For reference, we also indicate the Baryonic TF relation at $z \sim 0$ (McGaugh 2012), which includes the mass of the gas in the ordinate as well as the stellar mass.

Our new data reveal a remarkably similar slope to the previously measured Miller et al. relation at higher mass and similar redshifts (see Table 2 for the detailed comparison). Although aspects of the dwarf relation could suggest a somewhat steeper slope toward low mass than that inferred at high mass, this possible trend is marginal and requires more data for confirmation. More interesting is the distribution of the locally measured dwarfs relative to our sample at intermediate redshift. As seen in Figure 5, local galaxies lie mostly to the higher stellar mass (or rather slower velocity) side of the stellar mass TF relation. We will return to this topic in the next section.

We next consider the relation between stellar mass and halo mass, by converting $V_{2.2}$ to $V_{200}$ in Figure 6 using the relation measured by Reyes et al. (2012) with weak lensing. This relation is effectively calibrated at $\log \left(M_{*} / M_{\odot}\right)=9.0$, where $V_{2.2} / V_{200}=1.05$ and has a slope of $0.53 \pm 0.03$ (in terms of the relation of velocity ratio to stellar mass). To apply this in the low-mass regime of interest here requires extrapolating this relationship to stellar masses of $10^{7} M_{\odot}$, which clearly introduces some uncertainty. We note that preliminary abundance matching results of the Next Generation Virgo Survey (J. Grossauer et al., in preparation) in the dwarf regime are consistent with the extrapolation of the SHM relations from higher masses using abundance matching or weak lensing. Thus since the halo velocities in the Reyes et al. result are derived from weak-lensing as well, it is a fair hypothesis that the extrapolated relations are similar to what would be derived from the method conducted explicitly in the dwarf regime.

Assuming spherical symmetry, we further convert $V_{200}$ velocities (where the overdensity is 200 times the critical density, $\left.200 \rho_{\text {crit }}=M_{200} /(4 / 3) \pi R_{200}^{3}\right)$ to halo masses via the standard adopted relation where $\log M_{200} /\left[h^{-1} M_{\odot}\right]=$ $3 \log \left(V_{200}\left[\mathrm{~km} \mathrm{~s}^{-1}\right]\right) G^{-1}$. The gravitational constant $G$ here is $4.3 \times 10^{-6} \mathrm{kpc} M_{\odot}^{-1}\left(\mathrm{~km} \mathrm{~s}^{-1}\right)^{2}$. While abundance matching results and calibrations based on the latest weak lensing studies (e.g., Leauthaud et al. 2012; Behroozi et al. 2013) produce consistent results when compared to the stellar mass TF 


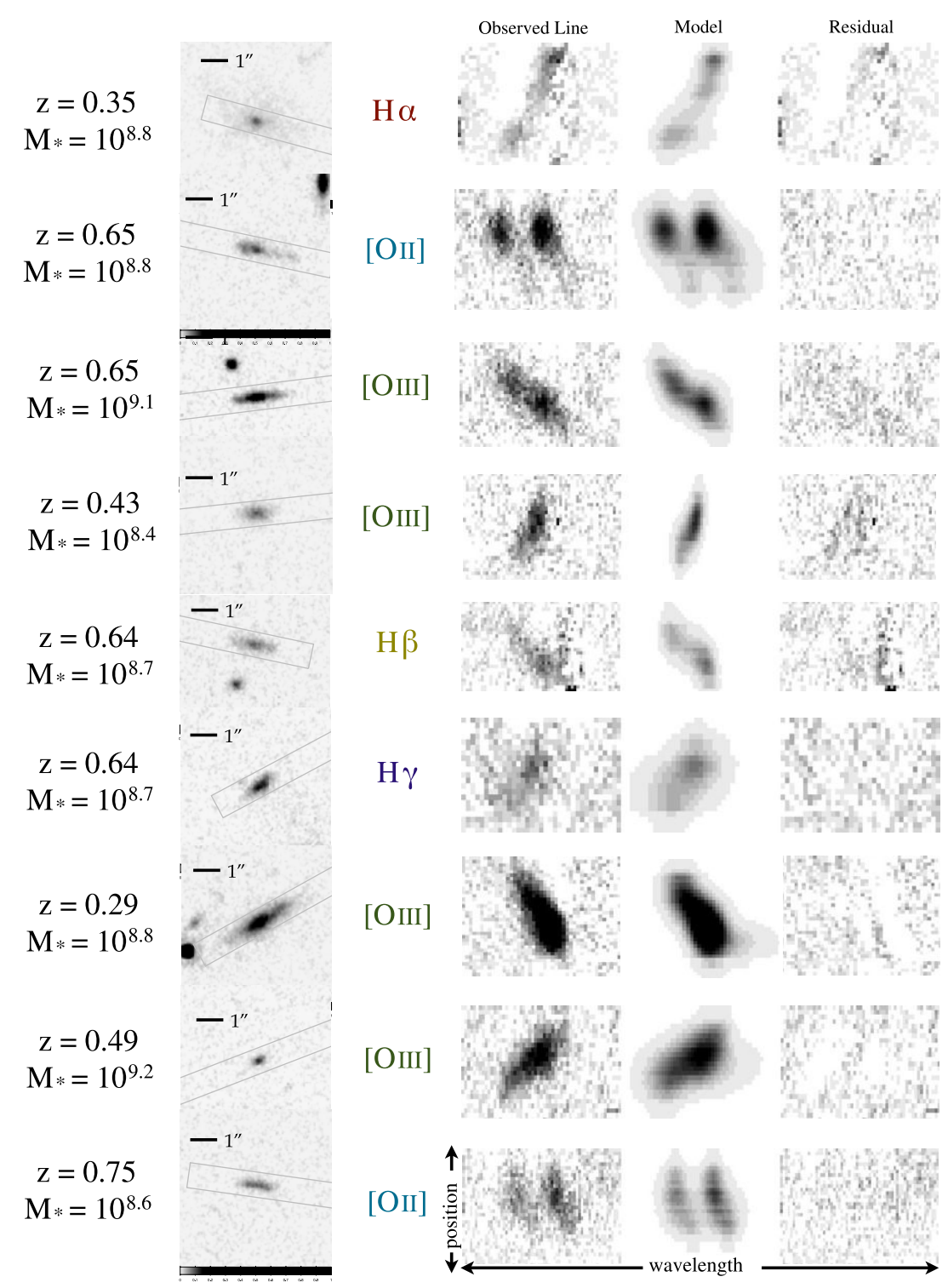

Hubble WFC3 F125W + Keck DEIMOS example line

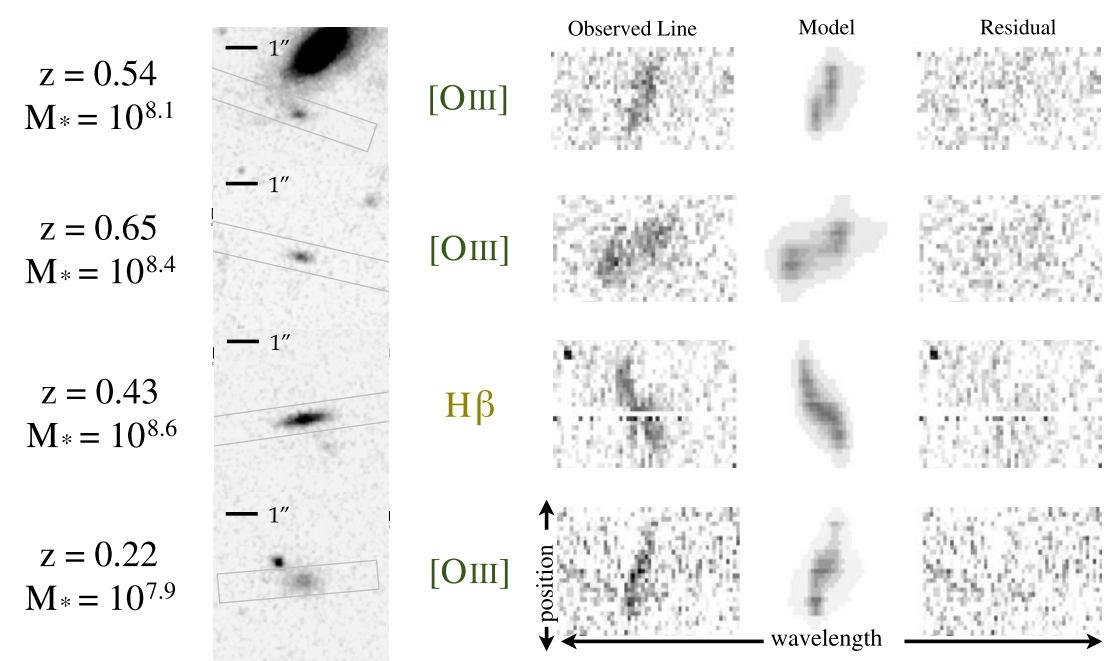

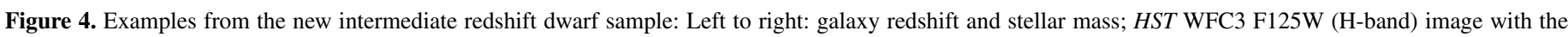

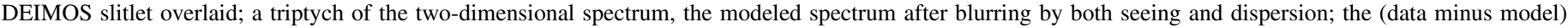
residual.

(A color version of this figure is available in the online journal.) 
Table 1

The Intermediate Redshift Dwarf Galaxy Sample

\begin{tabular}{|c|c|c|c|c|c|c|c|c|}
\hline R.A. & Decl. & $z^{\mathrm{a}}$ & $\sin (i)^{\mathrm{b}}$ & $\begin{array}{c}r_{s}^{\mathrm{c}} \\
(\mathrm{kpc})\end{array}$ & $\begin{array}{c}\log ^{\mathrm{d}} \\
\left(M_{*} / M_{\odot}\right)\end{array}$ & $\begin{array}{c}V_{2.2^{\mathrm{e}}} \\
\left(\mathrm{km} \mathrm{s}^{-1}\right)\end{array}$ & $\begin{array}{c}V_{1 \mathrm{kpc}}{ }^{\mathrm{f}} \\
\left(\mathrm{km} \mathrm{s}^{-1}\right)\end{array}$ & $\begin{array}{c}\log ^{\mathrm{g}} \\
\left(M_{h} / M_{\odot}\right)\end{array}$ \\
\hline \multicolumn{9}{|c|}{ Sample with Resolved Emission ( $N=41$; "Tully-Fisher sample") } \\
\hline 34.287564 & -5.1435433 & 0.64 & 0.94 & $1.04 \pm 0.31$ & $8.74 \pm 0.09$ & $97.77 \pm 3.82$ & $83.08 \pm 3.27$ & $10.79 \pm 0.03$ \\
\hline 34.303787 & -5.1458641 & 0.91 & 0.66 & $0.75 \pm 0.20$ & $8.32 \pm 0.12$ & $56.22 \pm 11.13$ & $45.29 \pm 8.97$ & $10.39 \pm 0.15$ \\
\hline 34.358524 & -5.1515666 & 0.35 & 0.66 & $0.41 \pm 0.25$ & $7.84 \pm 0.09$ & $16.01 \pm 9.71$ & $12.47 \pm 7.56$ & $9.36 \pm 0.39$ \\
\hline 34.398975 & -5.1525832 & 0.35 & 0.94 & $1.16 \pm 0.48$ & $8.79 \pm 0.07$ & $78.97 \pm 14.76$ & $72.21 \pm 13.46$ & $10.70 \pm 0.14$ \\
\hline 34.289186 & -5.1563347 & 0.57 & 0.91 & $0.77 \pm 0.29$ & $8.31 \pm 0.09$ & $31.35 \pm 15.67$ & $31.15 \pm 15.57$ & $9.80 \pm 0.35$ \\
\hline 34.359126 & -5.1571952 & 0.34 & 0.62 & $0.32 \pm 0.27$ & $7.29 \pm 0.14$ & $32.42 \pm 16.21$ & $32.30 \pm 16.15$ & $9.68 \pm 0.30$ \\
\hline 34.336092 & -5.1585409 & 0.31 & 0.52 & $0.40 \pm 0.36$ & $7.83 \pm 0.08$ & $17.29 \pm 3.26$ & $17.39 \pm 3.25$ & $9.56 \pm 0.13$ \\
\hline 34.489719 & -5.1596155 & 0.98 & 0.72 & $0.49 \pm 0.30$ & $8.64 \pm 0.14$ & $81.72 \pm 40.86$ & $81.06 \pm 40.53$ & $10.78 \pm 0.32$ \\
\hline 34.404135 & -5.1638168 & 0.43 & 0.87 & $0.68 \pm 0.15$ & $8.43 \pm 0.06$ & $50.21 \pm 5.89$ & $48.97 \pm 5.74$ & $10.20 \pm 0.09$ \\
\hline 34.310380 & -5.1642154 & 0.58 & 0.91 & $0.80 \pm 0.18$ & $8.54 \pm 0.13$ & $68.82 \pm 17.85$ & $68.36 \pm 17.73$ & $10.44 \pm 0.20$ \\
\hline 34.367527 & -5.1671357 & 0.65 & 0.92 & $0.66 \pm 0.35$ & $8.41 \pm 0.08$ & $81.89 \pm 30.77$ & $79.20 \pm 29.76$ & $10.47 \pm 0.27$ \\
\hline 34.440428 & -5.1676006 & 0.64 & 0.95 & $0.82 \pm 0.23$ & $8.74 \pm 0.09$ & $68.08 \pm 19.55$ & $46.02 \pm 13.00$ & $10.55 \pm 0.21$ \\
\hline 34.473897 & -5.1691046 & 0.31 & 0.77 & $0.35 \pm 0.28$ & $7.76 \pm 0.11$ & $33.26 \pm 12.27$ & $32.04 \pm 11.52$ & $9.71 \pm 0.25$ \\
\hline 34.446086 & -5.1700983 & 0.54 & 0.86 & $0.52 \pm 0.11$ & $8.15 \pm 0.15$ & $29.20 \pm 3.07$ & $28.60 \pm 3.01$ & $9.71 \pm 0.08$ \\
\hline 34.279085 & -5.1710765 & 0.35 & 0.47 & $0.63 \pm 0.22$ & $8.30 \pm 0.06$ & $40.32 \pm 11.63$ & $28.91 \pm 8.38$ & $10.38 \pm 0.16$ \\
\hline 34.350739 & -5.1712933 & 0.26 & 0.73 & $0.31 \pm 0.10$ & $7.25 \pm 0.11$ & $20.45 \pm 5.72$ & $16.88 \pm 4.85$ & $9.26 \pm 0.18$ \\
\hline 34.503690 & -5.1738860 & 0.43 & 0.34 & $0.57 \pm 0.34$ & $8.27 \pm 0.08$ & $18.78 \pm 5.41$ & $13.93 \pm 3.93$ & $10.09 \pm 0.18$ \\
\hline 34.432528 & -5.1760097 & 0.43 & 0.98 & $0.88 \pm 0.12$ & $8.75 \pm 0.09$ & $79.48 \pm 5.71$ & $72.14 \pm 5.17$ & $10.64 \pm 0.06$ \\
\hline 34.466202 & -5.1780329 & 0.29 & 0.97 & $1.07 \pm 0.17$ & $8.76 \pm 0.10$ & $58.70 \pm 2.42$ & $59.67 \pm 2.46$ & $10.46 \pm 0.03$ \\
\hline 34.429762 & -5.1785330 & 0.78 & 0.76 & $0.51 \pm 0.39$ & $8.96 \pm 0.06$ & $91.60 \pm 24.93$ & $55.58 \pm 15.92$ & $11.09 \pm 0.17$ \\
\hline 34.434409 & -5.1793119 & 0.78 & 0.96 & $0.72 \pm 0.20$ & $8.50 \pm 0.08$ & $77.75 \pm 45.98$ & $69.37 \pm 41.02$ & $10.46 \pm 0.40$ \\
\hline 34.517196 & -5.1793529 & 0.32 & 0.76 & $0.41 \pm 0.09$ & $7.89 \pm 0.07$ & $29.14 \pm 7.97$ & $28.12 \pm 7.69$ & $9.68 \pm 0.20$ \\
\hline 34.250846 & -5.1797838 & 0.35 & 0.74 & $0.34 \pm 0.29$ & $7.60 \pm 0.13$ & $48.38 \pm 5.91$ & $47.92 \pm 5.85$ & $9.92 \pm 0.09$ \\
\hline 34.415249 & -5.1820298 & 0.72 & 0.91 & $0.65 \pm 0.23$ & $8.50 \pm 0.08$ & $66.84 \pm 4.53$ & $66.25 \pm 4.48$ & $10.39 \pm 0.06$ \\
\hline 34.296140 & -5.1827143 & 0.21 & 0.96 & $0.58 \pm 0.20$ & $8.13 \pm 0.12$ & $47.67 \pm 3.51$ & $49.45 \pm 3.64$ & $9.94 \pm 0.06$ \\
\hline 34.362769 & -5.1829861 & 0.25 & 0.87 & $0.56 \pm 0.28$ & $8.03 \pm 0.09$ & $30.19 \pm 3.31$ & $29.87 \pm 3.28$ & $9.67 \pm 0.08$ \\
\hline 34.477748 & -5.1834746 & 0.34 & 0.84 & $0.38 \pm 0.21$ & $7.46 \pm 0.11$ & $45.24 \pm 1.52$ & $43.28 \pm 1.46$ & $9.75 \pm 0.03$ \\
\hline 34.423177 & -5.1861241 & 0.65 & 0.94 & $0.50 \pm 0.06$ & $7.95 \pm 0.08$ & $50.82 \pm 16.46$ & $43.36 \pm 13.67$ & $9.92 \pm 0.23$ \\
\hline 34.282862 & -5.1864030 & 0.45 & 0.73 & $0.35 \pm 0.09$ & $8.02 \pm 0.07$ & $50.73 \pm 13.39$ & $34.80 \pm 9.71$ & $10.11 \pm 0.15$ \\
\hline 34.520764 & -5.1871599 & 0.17 & 0.82 & $0.30 \pm 0.24$ & $7.06 \pm 0.27$ & $26.81 \pm 13.41$ & $28.01 \pm 14.00$ & $9.31 \pm 0.31$ \\
\hline 34.413980 & -5.1882020 & 0.88 & 0.65 & $0.38 \pm 0.26$ & $8.20 \pm 0.20$ & $31.35 \pm 8.27$ & $29.63 \pm 7.56$ & $9.96 \pm 0.09$ \\
\hline 34.523446 & -5.1899136 & 0.36 & 0.91 & $0.38 \pm 0.37$ & $7.40 \pm 0.21$ & $41.85 \pm 12.51$ & $41.70 \pm 11.88$ & $9.62 \pm 0.21$ \\
\hline 34.254774 & -5.1914894 & 0.65 & 0.70 & $0.84 \pm 0.29$ & $8.50 \pm 0.08$ & $55.40 \pm 15.34$ & $51.98 \pm 14.30$ & $10.45 \pm 0.19$ \\
\hline 34.359852 & -5.1916576 & 0.82 & 0.97 & $0.86 \pm 0.14$ & $8.66 \pm 0.14$ & $51.73 \pm 25.91$ & $43.39 \pm 21.73$ & $10.30 \pm 0.35$ \\
\hline 34.417074 & -5.1950333 & 0.65 & 0.89 & $0.55 \pm 0.27$ & $8.36 \pm 0.11$ & $37.47 \pm 6.58$ & $36.99 \pm 6.50$ & $9.95 \pm 0.13$ \\
\hline 34.370424 & -5.2035566 & 0.65 & 0.98 & $1.36 \pm 0.33$ & $8.80 \pm 0.11$ & $78.42 \pm 1.43$ & $75.90 \pm 1.39$ & $10.67 \pm 0.02$ \\
\hline 34.465397 & -5.2073517 & 0.59 & 0.73 & $0.44 \pm 0.23$ & $8.82 \pm 0.07$ & $54.11 \pm 14.78$ & $50.42 \pm 13.23$ & $10.64 \pm 0.13$ \\
\hline 34.446346 & -5.1498754 & 0.49 & 0.94 & $0.92 \pm 0.29$ & $9.24 \pm 0.12$ & $79.21 \pm 8.48$ & $78.91 \pm 7.87$ & $10.95 \pm 0.09$ \\
\hline 34.283345 & -5.1519078 & 0.65 & 0.98 & $1.08 \pm 0.13$ & $9.06 \pm 0.13$ & $87.92 \pm 8.62$ & $82.34 \pm 7.73$ & $10.96 \pm 0.08$ \\
\hline 34.392003 & -5.1590678 & 0.56 & 0.92 & $0.92 \pm 0.09$ & $8.94 \pm 0.07$ & $77.18 \pm 17.10$ & $65.16 \pm 14.63$ & $10.83 \pm 0.17$ \\
\hline 34.275351 & -5.1724717 & 0.41 & 0.88 & $0.74 \pm 0.44$ & $9.08 \pm 0.16$ & $77.91 \pm 5.11$ & $76.85 \pm 5.05$ & $10.96 \pm 0.05$ \\
\hline
\end{tabular}

Sample with Unresolved Emission $(N=18)$

\begin{tabular}{|c|c|c|c|c|c|c|c|c|}
\hline 34.452142 & -5.1527207 & 0.62 & $\cdots$ & $0.57 \pm 0.13$ & $8.29 \pm 0.10$ & $\cdots$ & $\cdots$ & $\cdots$ \\
\hline 34.490433 & -5.1591549 & 0.29 & $\cdots$ & $0.62 \pm 0.47$ & $8.58 \pm 0.09$ & $\cdots$ & $\cdots$ & $\cdots$ \\
\hline 34.333114 & -5.1617036 & 0.55 & $\cdots$ & $0.34 \pm 0.20$ & $8.45 \pm 0.13$ & $\cdots$ & $\cdots$ & $\cdots$ \\
\hline 34.501182 & -5.1717299 & 0.57 & $\cdots$ & $0.76 \pm 0.44$ & $8.88 \pm 0.12$ & $\cdots$ & $\cdots$ & $\ldots$ \\
\hline 34.269635 & -5.1725942 & 0.52 & $\cdots$ & $0.91 \pm 0.07$ & $8.36 \pm 0.08$ & $\cdots$ & $\cdots$ & $\cdots$ \\
\hline 34.321552 & -5.1734201 & 0.65 & $\cdots$ & $0.50 \pm 0.14$ & $8.45 \pm 0.08$ & $\cdots$ & $\cdots$ & $\cdots$ \\
\hline 34.393462 & -5.1748474 & 0.43 & $\ldots$ & $0.58 \pm 0.10$ & $8.33 \pm 0.08$ & $\cdots$ & $\cdots$ & $\ldots$ \\
\hline 34.338840 & -5.1827413 & 0.43 & $\cdots$ & $0.79 \pm 0.34$ & $8.83 \pm 0.07$ & $\cdots$ & $\cdots$ & $\ldots$ \\
\hline 34.344560 & -5.1913127 & 0.39 & $\cdots$ & $0.29 \pm 0.12$ & $7.95 \pm 0.11$ & $\cdots$ & $\cdots$ & $\cdots$ \\
\hline 34.496066 & -5.2060556 & 0.80 & $\cdots$ & $0.67 \pm 0.13$ & $8.72 \pm 0.17$ & $\cdots$ & $\cdots$ & $\ldots$ \\
\hline 34.266036 & -5.1594535 & 0.82 & $\cdots$ & $0.81 \pm 0.27$ & $8.35 \pm 0.16$ & $\cdots$ & $\cdots$ & $\cdots$ \\
\hline 34.338844 & -5.1653685 & 0.79 & $\cdots$ & $0.39 \pm 0.34$ & $8.50 \pm 0.10$ & $\cdots$ & $\cdots$ & $\cdots$ \\
\hline 34.481657 & -5.1674153 & 0.75 & $\cdots$ & $0.67 \pm 0.23$ & $8.66 \pm 0.06$ & $\cdots$ & $\cdots$ & $\ldots$ \\
\hline 34.370433 & -5.1823730 & 0.20 & $\cdots$ & $0.22 \pm 0.15$ & $7.57 \pm 0.16$ & $\cdots$ & $\cdots$ & $\cdots$ \\
\hline 34.481431 & -5.1833789 & 0.46 & $\cdots$ & $0.67 \pm 0.26$ & $8.95 \pm 0.09$ & $\cdots$ & $\cdots$ & $\cdots$ \\
\hline 34.514295 & -5.1839202 & 0.39 & $\cdots$ & $0.43 \pm 0.19$ & $8.23 \pm 0.09$ & $\cdots$ & $\cdots$ & $\ldots$ \\
\hline 34.341006 & -5.1872395 & 0.44 & $\cdots$ & $0.27 \pm 0.24$ & $8.10 \pm 0.08$ & $\cdots$ & $\cdots$ & $\cdots$ \\
\hline 34.375929 & -5.1970098 & 0.60 & $\cdots$ & $0.80 \pm 0.07$ & $8.38 \pm 0.16$ & $\cdots$ & $\cdots$ & $\cdots$ \\
\hline
\end{tabular}


Table 1

(Continued)

\begin{tabular}{|c|c|c|c|c|c|c|c|c|}
\hline R.A. & Decl. & $z^{\mathrm{a}}$ & $\sin (i)^{\mathrm{b}}$ & $\begin{array}{c}r_{s}^{\mathrm{c}} \\
(\mathrm{kpc})\end{array}$ & $\begin{array}{c}\log ^{\mathrm{d}} \\
\left(M_{*} / M_{\odot}\right)\end{array}$ & $\begin{array}{c}V_{2.2^{\mathrm{e}}} \\
\left(\mathrm{km} \mathrm{s}^{-1}\right)\end{array}$ & $\begin{array}{c}V_{1 \mathrm{kpc}^{\mathrm{f}}} \\
\left(\mathrm{km} \mathrm{s}^{-1}\right)\end{array}$ & $\begin{array}{c}\log ^{\mathrm{g}} \\
\left(M_{h} / M_{\odot}\right)\end{array}$ \\
\hline \multicolumn{9}{|c|}{ Sample with no Emission Detected $(N=23)$} \\
\hline 34.295579 & -5.1471624 & $(0.61)$ & $\cdots$ & $0.53 \pm 0.11$ & $8.73 \pm 0.12$ & $\cdots$ & $\cdots$ & $\cdots$ \\
\hline 34.403433 & -5.1479603 & $(0.38)$ & $\cdots$ & $0.34 \pm 0.10$ & $7.89 \pm 0.10$ & $\cdots$ & $\cdots$ & $\cdots$ \\
\hline 34.273705 & -5.1537420 & $(0.29)$ & $\cdots$ & $0.50 \pm 0.22$ & $8.07 \pm 0.10$ & $\cdots$ & $\cdots$ & $\cdots$ \\
\hline 34.422943 & -5.1545570 & $(0.58)$ & $\cdots$ & $0.73 \pm 0.26$ & $8.33 \pm 0.16$ & $\cdots$ & $\cdots$ & $\cdots$ \\
\hline 34.354658 & -5.1553817 & $(0.43)$ & $\cdots$ & $0.27 \pm 0.22$ & $8.20 \pm 0.07$ & $\cdots$ & $\ldots$ & $\ldots$ \\
\hline 34.396846 & -5.1563103 & $(0.43)$ & $\ldots$ & $0.48 \pm 0.06$ & $7.95 \pm 0.09$ & $\ldots$ & $\ldots$ & $\ldots$ \\
\hline 34.410996 & -5.1571028 & $(0.32)$ & $\cdots$ & $0.34 \pm 0.28$ & $7.85 \pm 0.09$ & $\ldots$ & $\ldots$ & $\ldots$ \\
\hline 34.327556 & -5.1592369 & $(0.22)$ & $\ldots$ & $0.25 \pm 0.20$ & $7.25 \pm 0.25$ & $\ldots$ & $\ldots$ & $\ldots$ \\
\hline 34.454441 & -5.1590304 & $(0.42)$ & $\cdots$ & $0.25 \pm 0.20$ & $7.90 \pm 0.27$ & $\ldots$ & $\ldots$ & $\ldots$ \\
\hline 34.509970 & -5.1648048 & $(0.66)$ & $\ldots$ & $0.36 \pm 0.11$ & $8.37 \pm 0.14$ & $\ldots$ & $\ldots$ & $\ldots$ \\
\hline 34.507877 & -5.1696592 & $(0.75)$ & $\ldots$ & $0.84 \pm 0.21$ & $8.62 \pm 0.15$ & $\ldots$ & $\ldots$ & $\ldots$ \\
\hline 34.305389 & -5.1736575 & $(0.28)$ & $\ldots$ & $0.38 \pm 0.09$ & $7.65 \pm 0.16$ & $\ldots$ & $\ldots$ & $\ldots$ \\
\hline 34.313890 & -5.1745230 & $(0.32)$ & $\ldots$ & $0.39 \pm 0.26$ & $7.81 \pm 0.09$ & $\ldots$ & $\ldots$ & $\ldots$ \\
\hline 34.398413 & -5.1746398 & $(0.76)$ & $\ldots$ & $0.59 \pm 0.43$ & $8.51 \pm 0.13$ & $\ldots$ & $\cdots$ & $\ldots$ \\
\hline 34.258658 & -5.1763573 & $(0.66)$ & $\cdots$ & $0.63 \pm 0.06$ & $8.73 \pm 0.10$ & $\ldots$ & $\cdots$ & $\ldots$ \\
\hline 34.447737 & -5.1786612 & $(0.52)$ & $\cdots$ & $0.46 \pm 0.09$ & $8.10 \pm 0.16$ & $\ldots$ & $\ldots$ & $\ldots$ \\
\hline 34.474809 & -5.1845825 & $(0.31)$ & $\ldots$ & $0.43 \pm 0.38$ & $7.86 \pm 0.11$ & $\ldots$ & $\ldots$ & $\ldots$ \\
\hline 34.495861 & -5.1862426 & $(0.27)$ & $\ldots$ & $0.26 \pm 0.09$ & $7.10 \pm 0.22$ & $\ldots$ & $\ldots$ & $\ldots$ \\
\hline 34.468164 & -5.1934894 & $(0.59)$ & $\cdots$ & $0.50 \pm 0.13$ & $8.05 \pm 0.07$ & $\cdots$ & $\cdots$ & $\cdots$ \\
\hline 34.453041 & -5.1942366 & $(0.39)$ & $\ldots$ & $0.39 \pm 0.13$ & $7.46 \pm 0.20$ & $\ldots$ & $\ldots$ & $\ldots$ \\
\hline 34.438733 & -5.1996580 & $(0.28)$ & $\cdots$ & $0.25 \pm 0.24$ & $8.13 \pm 0.11$ & $\ldots$ & $\ldots$ & $\ldots$ \\
\hline 34.507926 & -5.2060951 & $(0.64)$ & $\cdots$ & $0.78 \pm 0.50$ & $8.47 \pm 0.22$ & $\ldots$ & $\ldots$ & $\ldots$ \\
\hline 34.372178 & -5.2088495 & $(0.31)$ & $\ldots$ & $0.36 \pm 0.25$ & $7.88 \pm 0.12$ & $\ldots$ & $\ldots$ & $\ldots$ \\
\hline
\end{tabular}

Notes.

${ }^{a}$ Redshifts are spectroscopic except when in parentheses (photometric).

b Sine of inclination.

${ }^{\mathrm{c}}$ Exponential scale radius $(\mathrm{kpc})$.

${ }^{\mathrm{d}}$ Stellar mass in $\log \left(M_{*} / M_{\odot}\right)$.

${ }^{\text {e }}$ Rotation velocity $V_{2.2} \mathrm{~km} \mathrm{~s}^{-1}$.

${ }^{\mathrm{f}}$ Rotation velocity at $1 \mathrm{kpc}\left(\mathrm{km} \mathrm{s}^{-1}\right)$.

${ }^{\mathrm{g}}$ Halo mass inferred as described in Section 3, $\log \left(M_{h} / M_{\odot}\right)$.

Table 2

Stellar Mass TF Relation Fits

\begin{tabular}{|c|c|c|c|c|c|c|c|c|c|c|}
\hline$z$ Range & $\langle z\rangle$ & $N$ & $a^{\mathrm{a}}$ & $b^{\mathrm{b}}$ & $\sigma_{\text {int }, V^{\mathrm{c}}}$ & Med $\sigma_{V}{ }^{\mathrm{d}}$ & $\mathrm{rms}_{V} \mathrm{e}^{\mathrm{e}}$ & $\sigma_{\text {int }, M^{\mathrm{f}}}$ & Med $\sigma_{M}^{\mathrm{g}}$ & $\mathrm{rms}_{M}{ }^{\mathrm{h}}$ \\
\hline \multicolumn{11}{|c|}{$M_{*}>10^{9} M_{\odot}$ Relation (Miller et al. 2011): $M_{*}$ vs. $V\left(r_{2.2}\right)$} \\
\hline $0.2<z \leqslant 1.3$ & 0.64 & 129 & $1.718 \pm 0.415$ & $3.869 \pm 0.193$ & 0.058 & 0.022 & 0.083 & 0.224 & 0.091 & 0.323 \\
\hline \multicolumn{11}{|c|}{ Dwarf Mass Relation (This Work): $M_{*}$ vs. $V\left(r_{2.2}\right)$} \\
\hline $0.2<z \leqslant 1.0$ & 0.64 & 41 & $0.566 \pm 0.476$ & $4.347 \pm 0.623$ & 0.064 & 0.078 & 0.122 & 0.375 & 0.095 & 0.532 \\
\hline
\end{tabular}

Notes.

a $\mathrm{y}$-int in $M_{*} / M_{\odot}$ dex assuming scatter in $V / \mathrm{km} \mathrm{s}^{-1}$ dex.

b Slope assuming scatter in $V / \mathrm{km} \mathrm{s}^{-1}$ dex.

c Internal scatter in $V / \mathrm{km} \mathrm{s}^{-1}$ dex.

${ }^{\mathrm{d}}$ Median velocity error in $V / \mathrm{km} \mathrm{s}^{-1}$ dex.

e Total scatter in $V / \mathrm{km} \mathrm{s}^{-1}$ dex.

${ }^{\mathrm{f}}$ Internal scatter in $M_{*} / M_{\odot}$ dex.

g Median stellar mass error in $M_{*} / M_{\odot}$ dex.

h Total scatter in $M_{*} / M_{\odot}$ dex.

relation above masses of $10^{9} M_{\odot}$, there is a clear divergence in the dwarf regime of these relations in Figure 6. We discuss the implications and uncertainties of this result in the next section.

\section{DISCUSSION}

\subsection{Is the Local Group Population Representative?}

Figure 5 shows a striking effect, namely that local galaxies appear to be drawn from a particular subset of the stellar mass
TF relation defined by our larger sample. It seems unlikely that this is an evolutionary effect (relating, for example, to changes in the dark matter density profiles or effect of baryonic feedback) given there is no obvious trend with redshift within our sample itself. Otherwise this would result in the curious conclusion that the relation between baryons and dark matter becomes tighter over time when non-linear exchanges dominate. A more likely explanation is that we are sensitive to sources at intermediate redshift that are not typically found locally. For 


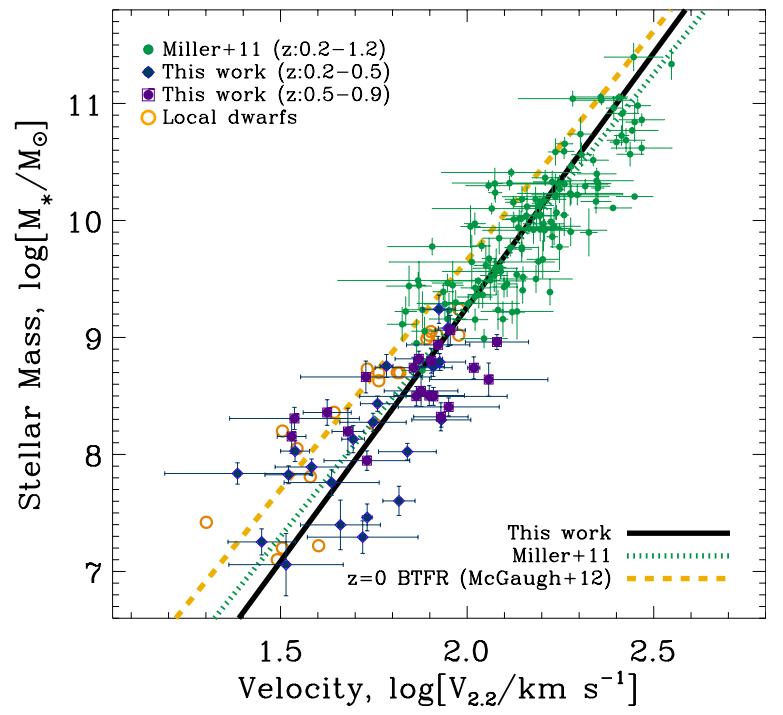

Figure 5. Stellar mass Tully-Fisher (TF) relation: the new sample of lowmass galaxies is shown according to two redshift intervals: blue diamonds $(0.2<z<0.5)$ and purple squares $(0.5<z<0.9)$. A more massive sample spanning the full redshift range is shown with green points (Miller et al. 2011) and the Local Group dwarf population at the targeted mass range, including the Magellanic Clouds, is plotted with golden rings. Lines represent best fits to the dwarf stellar mass TF relation (solid black), the intermediate redshift sample (Miller et al. 2011; dotted green), and the local Baryonic TF relation as determined by McGaugh (2012; yellow dashed).

(A color version of this figure is available in the online journal.)

example, low-mass galaxies generally may have a wider range of gas fractions which could explain the direction of the offset and increased scatter. Clearly we are biased in our survey toward dwarf galaxies with higher gas fractions that allow the tracing of extended emission lines for our kinematic analysis. Also the field of selection within the DEIMOS mask samples on average lower density environments compared to the group environment locally. This may proportionally affect dwarfs more than higher mass galaxies due to a satellite effect: gas is lost to interactions with other satellites and the host galaxy, whereby in the field and in voids dwarf galaxies are more likely to keep their gas. This is also why, throughout this paper, we do not consider local dE/dS0 populations. Although dark-matter-dominated, to utilize these galaxies, we would need to additionally convert their stellar velocity dispersion measurements to circularized rotational velocities. Such galaxies have lost their interstellar media and a distinct population from the star-forming dwarfs that form the basis of the present study. It would also be difficult to robustly compare such sources with the abundance matching methods given that most of their baryonic content is indistinguishable from the inter-cluster or inter-group medium to which it was lost. These environmental themes and a more direct comparison of $\mathrm{dE} / \mathrm{dS} 0$ galaxies with star-forming dwarfs will be explored further in future work.

Taking this argument further, it is interesting to speculate what role gas fractions may play in reconciling the abundance matching discrepancy in Figure 6. The rank order for matching halos to observed mass functions could vary widely moving into the dwarf regime with the inclusion of gas mass. Separately, could including gas and realistic feedback prescriptions in the simulations used to construct halo trees for abundance matching have an appreciable effect toward reconciling the offset? Neither of the SHM relations being compared for consistency in Figure 6 take full account of the effect of gas. Unfortunately, future

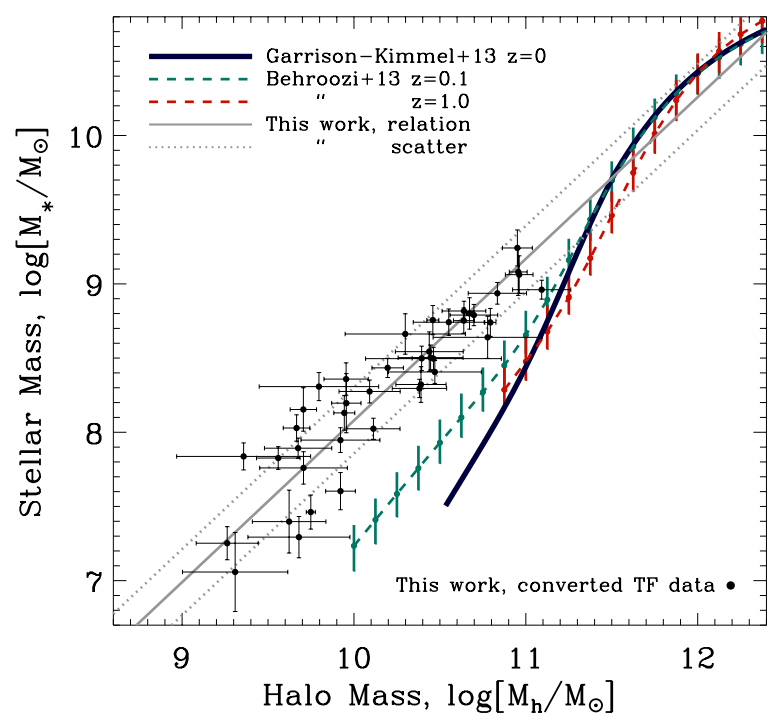

Figure 6. Stellar-to-halo mass relation according to the abundance matching curves of Garrison-Kimmel et al. (2013; navy curve at $z=0$ ) and Behroozi et al. (2013; aqua and red curves at $z=0.1$ and $z=1.0$, respectively) derived in $\Lambda \mathrm{CDM}$, compared to that inferred for our sample of intermediate redshift dwarf galaxies by converting the measured $V_{2.2}$ velocities in the stellar mass Tully-Fisher relation to halo masses using the procedure discussed in Section 3. Black points represent the data, the solid gray line is the best-fit relation, and the scatter is indicated with dashed gray lines.

(A color version of this figure is available in the online journal.)

progress on this front will be hindered by the difficulty in measuring gas masses in the dwarf regime as well as at higher- $z$ where they appear to be greater on average than at low redshift (Tacconi et al. 2010).

\subsection{The Role of Feedback? New Dark Matter Physics?}

Implementing episodic supernova feedback in hydrodynamical simulations (Governato et al. 2012) has been successful in flattening dark matter density profiles of halos over time. This may even resolve a related, long-standing difficulty whereby simulations predicting inner density profiles that are too "cuspy" (Pontzen \& Governato 2012). Although this effect can be reproduced in both adaptive mesh refinement simulations (Teyssier et al. 2013), and smoothed particle hydrodynamics, other authors have had difficulty reproducing the result in alternative prescriptions of the same feedback process. While this action may work to alleviate the discrepancy in Figure 6 if the redistribution of matter was effective to $2.2 \mathrm{kpc}$, even efficient feedback may be insufficient to remove the discrepancy by the required amount (Sawala et al. 2013; Munshi et al. 2013). In fact, no amount of feedback has been effective at significantly flattening inner density profiles below stellar masses of $10^{7} M_{\odot}$ (Governato et al. 2012). Thus, if the offset observed in Figure 5 can be shown to be present in dwarfs $\sim 1$ dex lower in stellar mass, it would present a fundamental problem for arguments that the entire effect is due to baryonic effects. Additionally, there are arguments that the energy of the necessary feedback for this effect exceeds what would likely occur over the typical star-formation history of most dwarfs (Garrison-Kimmel et al. 2013).

Alternatively, a combination of early feedback effects with ram pressure stripping and tidal heating by both the host halo and disk appear to extract enough energy from the gravitational potential of the host to reproduce the observations in some examples (Arraki et al. 2013; Zolotov et al. 2012; Brooks 
et al. 2013). In future work we hope to better ascertain how environmental effects may be responsible for our observed trends by determining whether targeted dwarfs are isolated or likely satellites. If most of the dwarfs are isolated, as suspected given their inferred gas-rich state, then it will be difficult to use this mechanism to explain Figure 6.

Ultimately, a more fundamental adjustment to the dark matter model could be required (Weinberg et al. 2013). Alternative dark matter models that were not fully explored before the last decade of consensus around CDM include warm dark matter models (e.g., Benson 2012; Lovell et al. 2012), various selfinteracting dark matter models (Spergel \& Steinhardt 2000; Rocha et al. 2013; Peter et al. 2013), and flavor-mixed dark matter (Medvedev 2001, 2012). Any of these may potentially hold the key in resolving observed tensions in the CDM predictions for galaxy formation and evolution, however work has only just begun exploring these alternative models in detail.

\subsection{The Utility of the Stellar to Halo Mass Relation}

Figure 6 is difficult to interpret without acknowledging CDM simulations generate too much substructure. When galaxies are matched to halos from the observed stellar mass functions, they are placed in halos which are too large to be consistent with the observed fiducial rotation velocities related to their stellar mass. This discrepancy increases as the (mis-)matching propagates into the dwarf galaxy regime.

We recognize that the utility of Figure 6 in the dwarf regime relies on certain aspects of the CDM halo paradigm for us to convert observed velocities at observable radii to those of the unobservable halo. Several effects could explain the discrepancy in Figure 6, the most obvious of which is that the linear extrapolation of the Reyes et al. (2012) relation (connecting $V_{2.2}$ to $V_{200}$ ) to the dwarf regime could be inaccurate, as discussed in Section 4. As discussed in Section 3.2, if the stellar mass estimates are underestimated from the use of inappropriate stellar population synthesis models or unaccounted for emission flux in the broad band data, then such underestimates would exacerbate the discrepancy in Figure 6. Most importantly, the gas mass should be accounted for to create a "Baryonic" TF consistency check; this might reconcile the offset seen in Figure 5 and affect the abundance matching method currently based only on the stellar mass distribution. Finally, only when such caveats can be laid to rest might we seriously consider powerful feedback effects which would modify dark matter density profiles out to $2.2 \mathrm{kpc}$ in cosmological simulations or dark matter physics beyond the standard $\Lambda$ CDM paradigm.

\subsection{A More Direct Observational Test}

Noting the considerable uncertainties in the interpretation of Figure 6, a more direct test would be to extract quantities such as $V_{2.2}$ from halos within the cosmological simulations, in order to conduct a comparison in the observed space, i.e., Figure 5. Ideally, the abundance matching curve with its scatter would not be extracted in terms of the stellar mass TF relation, but rather the full baryonic TF relation with the inclusion of gas mass. Not accounting for the effects of gas in a comprehensive manner may ultimately lead to the mismatch we see between simulated CDM predictions and observations in each context: from early versions of the "missing satellite problem," to the "Too Big to Fail" framing within the Milky Way, to the semiempirical curves of abundance matching diverging away from the featureless TF relations. A more careful comparison, one which includes gas rather than stellar mass alone, is needed to explore these ideas further and will be addressed in future work.

S.H.M. thanks the Rhodes Trust, the British Federation of Women Graduates, the sub-department of Astrophysics and New College at the University of Oxford, the University of California, Riverside, and the California Institute of Technology for supporting her work. We thank the referee for his helpful comments which have improved the quality of this work. The spectroscopic data was secured with the W. M. Keck Observatory on Mauna Kea. We thank the observatory staff for their dedication and support. The authors recognize and acknowledge the cultural role and reverence that the summit of Mauna Kea has always had with the indigenous Hawaiian community, and we are most fortunate to have the opportunity to conduct observations from this mountain.

Facilities: Keck:II (DEIMOS), HST (WFC3/IR).

\section{REFERENCES}

Arraki, K. S., Klypin, A., More, S., \& Trujillo-Gomez, S. 2013, MNRAS Behroozi, P. S., Conroy, C., \& Wechsler, R. H. 2010, ApJ, 717, 379 Behroozi, P. S., Wechsler, R. H., \& Conroy, C. 2013, ApJ, 770, 57 Benson, A. 2012, NewA, 17, 175

Bertin, E., \& Arnouts, S. 1996, A\&AS, 117, 393

Boylan-Kolchin, M., Bullock, J. S., \& Kaplinghat, M. 2011, MNRAS, 415, L40 Brammer, G. B., van Dokkum, P. G., \& Coppi, P. 2008, ApJ, 686, 1503 Brooks, A. M., Kuhlen, M., Zolotov, A., \& Hooper, D. 2013, ApJ, 765, 22 Bruzual, G., \& Charlot, S. 2003, MNRAS, 344, 1000

Bullock, J. S., Dekel, A., Kolatt, T. S., et al. 2001, ApJ, 555, 240

Bullock, J. S., Kravtsov, A. V., \& Weinberg, D. H. 2000, ApJ, 539, 517

Calzetti, D., Armus, L., Bohlin, R. C., et al. 2000, ApJ, 533, 682

Chabrier, G. 2003, PASP, 115, 763

Chilingarian, I. V., Cayatte, V., \& Bergond, G. 2008, MNRAS, 390, 906

Cirasuolo, M., McLure, R. J., Dunlop, J. S., et al. 2010, MNRAS, 401, 1166

Coelho, P., Bruzual, G., Charlot, S., et al. 2007, MNRAS, 382, 498

Conroy, C., Prada, F., Newman, J. A., et al. 2007, ApJ, 654, 153

Courteau, S. 1997, AJ, 114, 2402

De Rijcke, S., Prugniel, P., Simien, F., \& Dejonghe, H. 2006, MNRAS, 369,1321

Diemand, J., Kuhlen, M., \& Madau, P. 2007, ApJ, 667, 859

Diemand, J., Kuhlen, M., Madau, P., et al. 2008, Natur, 454, 735

Dutton, A. A., Conroy, C., van den Bosch, F. C., Prada, F., \& More, S. 2010, MNRAS, 407, 2

Eke, V. R., Baugh, C. M., Cole, S., Frenk, C. S., \& Navarro, J. F. 2006, MNRAS, 370,1147

Font, A. S., Benson, A. J., Bower, R. G., et al. 2011, MNRAS, 417, 1260

Furusawa, H., Kosugi, G., Akiyama, M., et al. 2008, in ASP Conf. Ser. 399, Panoramic Views of Galaxy Formation and Evolution, ed. T. Kodama, T. Yamada, \& K. Aoki (San Francisco, CA: ASP), 131

Garrison-Kimmel, S., Rocha, M., Boylan-Kolchin, M., Bullock, J. S., \& Lally, J. 2013, MNRAS, 433, 3539

Geha, M., Blanton, M. R., Masjedi, M., \& West, A. A. 2006, ApJ, 653, 240

Governato, F., Zolotov, A., Pontzen, A., et al. 2012, MNRAS, 422, 1231

Grogin, N. A., Kocevski, D. D., Faber, S. M., et al. 2011, ApJS, 197, 35

Klypin, A., Kravtsov, A. V., Valenzuela, O., \& Prada, F. 1999, ApJ, 522, 82

Koekemoer, A. M., Faber, S. M., Ferguson, H. C., et al. 2011, ApJS, 197, 36

Kormendy, J., \& Bender, R. 2012, ApJS, 198, 2

Kriek, M., van Dokkum, P. G., Labbé, I., et al. 2009, ApJ, 700, 221

Leauthaud, A., Tinker, J., Bundy, K., et al. 2012, ApJ, 744, 159

Lovell, M. R., Eke, V., Frenk, C. S., et al. 2012, MNRAS, 420, 2318

Mandelbaum, R., Seljak, U., Kauffmann, G., Hirata, C. M., \& Brinkmann, J. 2006, MNRAS, 368, 715

McGaugh, S. S. 2012, AJ, 143, 40

Medvedev, M. V. 2001, in AIP Conf. Proc. Vol. 586, Relativistic Astrophysics, ed. J. C. Wheeler \& H. Martel (Melville, NY: AIP), 149

Medvedev, M. V. 2012, in APS April Meeting Abstracts, G7007

Miller, S. H., Bundy, K., Sullivan, M., Ellis, R. S., \& Treu, T. 2011, ApJ, 741,115

Miller, S. H., Ellis, R. S., Sullivan, M., et al. 2012, ApJ, 753, 74

Miller, S. H., Sullivan, M., \& Ellis, R. S. 2013, ApJL, 762, L11

Moore, B., Ghigna, S., Governato, F., et al. 1999, ApJL, 524, L19

More, S., van den Bosch, F. C., Cacciato, M., et al. 2011, MNRAS, 410, 210 
Moster, B. P., Somerville, R. S., Maulbetsch, C., et al. 2010, ApJ, 710, 903

Munshi, F., Governato, F., Brooks, A. M., et al. 2013, ApJ, 766, 56

Navarro, J. F., Frenk, C. S., \& White, S. D. M. 1997, ApJ, 490, 493

Newman, J. A., Cooper, M. C., Davis, M., et al. 2013, ApJS, 208, 5

Oh, S.-H., Brook, C., Governato, F., et al. 2011, AJ, 142, 24

Peng, C. 2010, BAAS, 42, 578

Peter, A. H. G., Rocha, M., Bullock, J. S., \& Kaplinghat, M. 2013, MNRAS, 430, 105

Pizagno, J., Prada, F., Weinberg, D. H., et al. 2007, AJ, 134, 945

Pontzen, A., \& Governato, F. 2012, MNRAS, 421, 3464

Reyes, R., Mandelbaum, R., Gunn, J. E., et al. 2012, MNRAS, 425, 2610

Rocha, M., Peter, A. H. G., Bullock, J. S., et al. 2013, MNRAS, 430, 81

Sawala, T., Frenk, C. S., Crain, R. A., et al. 2013, MNRAS, 431, 1366
Seljak, U. 2002, MNRAS, 334, 797

Spergel, D. N., \& Steinhardt, P. J. 2000, PhRvL, 84, 3760

Springel, V., Wang, J., Vogelsberger, M., et al. 2008, MNRAS, 391, 1685

Stark, D. V., McGaugh, S. S., \& Swaters, R. A. 2009, AJ, 138, 392

Tacconi, L. J., Genzel, R., Neri, R., et al. 2010, Natur, 463, 781

Teyssier, R., Pontzen, A., Dubois, Y., \& Read, J. I. 2013, MNRAS, 429, 3068

Tollerud, E. J., Bullock, J. S., Strigari, L. E., \& Willman, B. 2008, ApJ, 688, 277

Weinberg, D. H., Bullock, J. S., Governato, F., Kuzio de Naray, R., \& Peter, A. H. G. 2013, arXiv:1306.0913

Wuyts, S., Förster Schreiber, N. M., Lutz, D., et al. 2011, ApJ, 738, 106

Zolotov, A., Brooks, A. M., Willman, B., et al. 2012, ApJ, 761, 71 